\title{
ESTABILIZACIÓN DE EÓLICA MARINA FLOTANTE MEDIANTE CONTROL DE FLUJO DE AIRE EN COLUMNAS DE AGUA OSCILANTE
}

\author{
Fares M'zoughi ${ }^{1}$, Payam Aboutalebi ${ }^{1}$, Izaskun Garrido ${ }^{1}$, Aitor J. Garrido ${ }^{1}$ and Manuel De La Sen ${ }^{2}$ \\ ${ }^{1}$ Automatic Control Group-ACG, Institute of Research and Development of Processes-IIDP, \\ Department of Automatic Control and Systems Engineering, Faculty of Engineering of Bilbao, \\ University of the Basque Country-UPV/EHU, Po Rafael Moreno no3, 48013 Bilbao, Spain \\ fares.mzoughi@ehu.eus; payam.aboutalebi@ehu.eus; izaskun.garrido@ehu.eus; aitor.garrido@ehu.eus \\ ${ }^{2}$ Automatic Control Group-ACG, Institute of Research and Development of Processes-IIDP, \\ Department of Electricity and Electronics. Faculty of Science and Technology, University of the Basque \\ Country_UPV/EHU, Sarriena s/n 48940 Leioa, Spain \\ manuel.delasen@ehu.eus
}

\begin{abstract}
Resumen
En este artículo se presenta un nuevo tipo de control aplicado a una plataforma flotante multipropósito tipo barcaza capaz de aprovechar la energía eólica y undimotriz de manera simultánea. Además, los captadores de energía undimotriz se componen de columnas de agua oscilante (OWC) que ayudarán a estabilizar la plataforma y la turbina eólica flotante (FOWT) reduciendo el desplazamiento longitudinal de la parte superior de la torre. Para ello se implementa una estrategia de control de flujo de aire actuando sobre las válvulas de la cámara de captura de los dispositivos OWC. El estudio comparativo entre una FOWT estándar y la FOWT sobre la plataforma dotada de dispositivos controlados OWC propuesta, muestra una considerable mejora en la estabilidad del sistema.
\end{abstract}

Palabras clave: control del flujo de aire; plataforma tipo barcaza; turbina eólica marina flotante; columna de agua oscilante; energía undimotriz; energía eólica.

\section{INTRODUCCIÓN}

En los últimos años, el impulso a las renovables está impulsando el uso de turbinas eólicas marinas como plataformas multipropósito para un mejor uso, eficiencia y reducción de costes.

La plataforma más estudiada son las plataformas mixtas que combinan turbinas eólicas y convertidores de energía de las olas (WEC) para aprovechar la energía undimotriz y eólica [1-3]. En este artículo se propone el uso de OWC con una turbina eólica marina flotante tipo barge $y$ se han mostrado resultados prometedores. J.M. Kluger y col. [4] investigó el uso de una matriz WEC con FOWT tipo spar del Laboratorio Nacional de Energía Renovable (NREL) llamado OC3-Hywind FOWT. Posteriormente, A. Slocum et al. [5] discutió el uso de absorvedores puntuales internos y externos a una FOWT. M. Kamarlouei y col. [6] llegó a la conclusión de que la instalación de una matriz de WEC puede disminuir los movimientos de la plataforma de la FOWT en elevación y cabeceo. Sin embargo, los enfoques introducidos no han utilizado OWC en plataformas FOWT tipo barcaza.

Aunque las OWC se han aplicado en diferentes FOWT para disminuir desplazamientos, especialmente aquellas de tipo spar, aún no se ha analizado la aplicación de OWC para la estabilización de FOWT tipo barcaza. Este trabajo tiene como objetivo combinar una turbina eólica marina flotante con una columna de agua oscilante para aprovechar tanto la energía undimotriz como la eólica y estudiar la estabilización de la FOWT utilizando los OWC. La FOWT considerada es el aerogenerador NREL de $5 \mathrm{MW}$ montado en la plataforma ITI Energy tipo barcaza. La barcaza ITI Energy es un concepto que fue desarrollado por el Departamento de Arquitectura Naval e Ingeniería Marina de las Universidades de Glasgow y Strathclyde a través de un contrato con ITI Energy (consulte [7] para obtener una explicación detallada sobre este tema). La idea es integrar OWCs dentro de la barcaza ITI Energy para ayudar a reducir los movimientos no deseados de la plataforma. Esto se puede lograr controlando las válvulas de aire de cada OWC usando una estrategia de control de flujo de aire [8-10] para ajustar el flujo de aire y la presión dentro de las cámaras de captura. 
El resto del artículo está organizado de la siguiente manera: En la Sección 2 se presenta el Modelo FOWT y sus ecuaciones. Luego, se propone en la Sección 3 un control de flujo de aire, implementado para regular el flujo de aire y la presión en las cámaras con el fin de estabilizar la FOWT. En la Sección 4 se realiza un estudio comparativo del FOWT entre la barcaza ITI Energy estándar y la barcaza basada en OWC. Finalmente, la Sección 5 cierra el documento con algunas observaciones.

\section{MODELO DE PLATAFORMA FOWT BASADO EN OWC}

La turbina a considerar es de tres palas, de velocidad variable y de paso controlado con un diámetro de rotor de $126 \mathrm{~m}$. Las palas accionan un generador instalado en la parte superior de la torre en la góndola a una altura de buje de $90 \mathrm{~m}$. La turbina eólica está montada sobre una plataforma tipo barcaza lastrada, amarrada por líneas de catenaria para reducir la deriva y mejorar la estabilidad. Las especificaciones detalladas de la turbina eólica de 5 MW y la plataforma de barcaza ITI se pueden encontrar en [11], aunque algunos parámetros básicos se proporcionan en la Tabla 1.

Tabla 1: Parámetros del aerogenerador NREL 5 MW y la plataforma de barcaza ITI.

\begin{tabular}{|l|l|}
\hline Feature & Value \\
\hline Potencia nominal & $5 \mathrm{MW}$ \\
Control & Velocidad variable \\
Velocidad del viento & $3 \mathrm{~m} / \mathrm{s}, 11.4 \mathrm{~m} / \mathrm{s}, 25 \mathrm{~m} / \mathrm{s}$ \\
cut-in, rated, cut-out & \\
Velocidad del rotor & $6.9 \mathrm{rpm}, 12.1 \mathrm{rpm}$ \\
Cut-in, rated & \\
Masa de la torre & $347,460 \mathrm{~kg}$ \\
$\begin{array}{l}\text { Diámetro del rotor } \\
\text { Altura del buje }\end{array}$ & $126 \mathrm{~m}$ \\
Tamaño de la & $90 \mathrm{~m}$ \\
plataforma de & $40 \mathrm{~m} \mathrm{x} 10 \mathrm{~m}$ \\
Masa de la plataforma & $5,452,000 \mathrm{~kg}$ \\
Número de líneas de & 8 \\
amarre delanda & $150 \mathrm{~m}$ \\
Profundidad del ancla & $130.4 \mathrm{~kg} / \mathrm{m}$ \\
Diámetro de la línea \\
Densidad de masa \\
lineal
\end{tabular}

Según numerosos estudios, a pesar de que las plataformas flotantes tienen un gran desplazamiento en la vertical, es bien sabido que el movimiento de cabeceo de la barcaza que más contribuye a la flexión de la torre [12]. Este cabeceo de la barcaza alrededor del eje Y, afecta el desplazamiento de la parte superior de la torre en la dirección del eje $\mathrm{X}$, por lo que el desplazamiento longitudinal también es muy importante en la estabilidad de FOWT. Por lo tanto, solo habilitamos el modo de cabeceo. Se ha demostrado que este enfoque es suficientemente eficaz para el control de la vibración estructural de los FOWT [13-15]. Por lo tanto, el modelo del sistema considera dos modos de la turbina eólica, que son el movimiento de inclinación de la plataforma y el modo de flexión de la torre en primer lugar. Además, el trabajo de este artículo se centra en las dinámicas de vibración de las FOWT que están más relacionadas con las olas. Asimismo, se ha omitido el efecto viento sobre la turbina con el fin de obtener un modelo simplificado y diseñar el control adecuado.

\subsection{MODELO DINÁMICO DE UN FOWT BASADO EN OWC}

Para el FOWT de tipo barcaza, los grados de libertad más importantes que afectan las cargas de la torre de la estructura de la turbina eólica son el movimiento de inclinación de la plataforma y el primer modo de flexión longitudinal de la torre [16,17]. Por lo tanto, ambos se utilizan en el diseño del modelo simplificado de turbina eólica de orden reducido, y su esquema se muestra en la Figura 1.

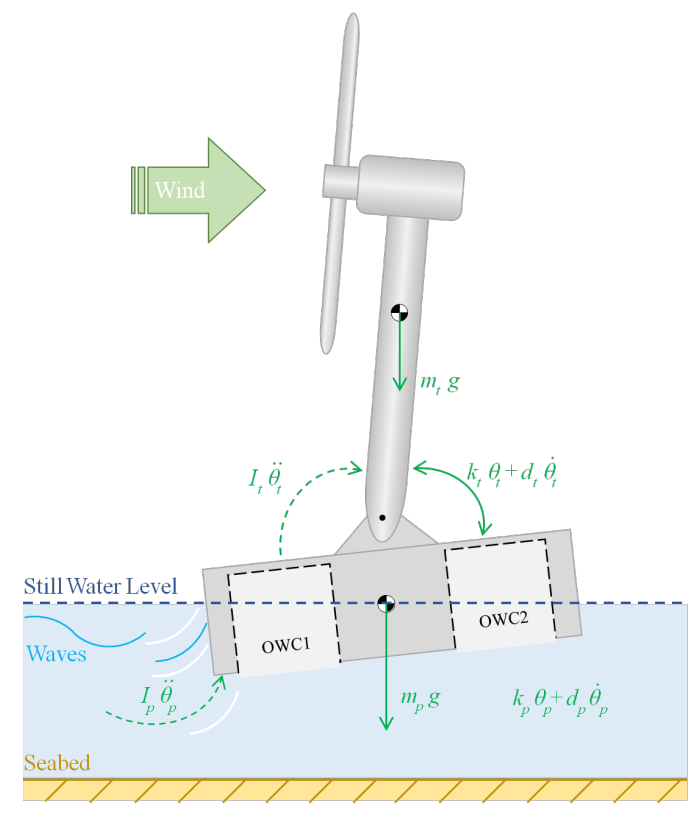

Figura 1: Modelo simplificado de turbina eólica marina flotante.

Las ecuaciones de Euler-Lagrange de un sistema no conservador con $\mathrm{n}$ coordenadas generalizadas se describen mediante (1) y (2):

$$
\begin{aligned}
\frac{d}{d t}\left(\frac{\partial L}{\partial \dot{q}_{i}}\right)-\frac{\partial L}{\partial q_{i}} & =Q_{i} \quad(i=1,2, \cdots, n) \\
L & =T-V
\end{aligned}
$$


donde $T$ y $V$ son la energía cinética total y la energía potencial total del sistema, respectivamente. $L$ es el operador de Lagrange. La energía cinética total y la energía potencial total de la turbina eólica flotante tipo barcaza se pueden expresar como:

$$
\begin{aligned}
T & =\frac{1}{2} I_{t} \dot{\theta}_{t}^{2}+\frac{1}{2} I_{p} \dot{\theta}_{p}^{2} \\
V & =\frac{1}{2} k_{t}\left(\theta_{t}-\theta_{p}\right)^{2}+\frac{1}{2} k_{p} \theta_{p}^{2} \\
& +m_{t} g R_{t} \cos \theta_{t}-m_{p} g R_{p} \cos \theta_{p}
\end{aligned}
$$

donde $k$ es el coeficiente de rigidez del resorte equivalente, $\theta$ es el ángulo de rotación alrededor del eje vertical $\mathrm{z}, I$ es el momento de inercia alrededor del centro de masa. $m$ es la masa, $g$ es la aceleración gravitacional y $R$ es la distancia desde el centro de masa hasta la sujeción de la torre. Los subíndices $p \mathrm{y}$ $t$ representan la plataforma y la torre, respectivamente.

Las fuerzas generalizadas consisten en la fuerza de amortiguación y las cargas de viento y oleaje externas expresadas como:

$$
\left\{\begin{aligned}
Q_{\theta_{t}}= & -d_{t}\left(\dot{\theta}_{t}-\dot{\theta}_{p}\right)+M_{\text {wind }} \\
Q_{\theta_{p}}= & -d_{p} \dot{\theta}_{p}+d_{t}\left(\dot{\theta}_{t}-\dot{\theta}_{p}\right)+M_{\text {wave }} \\
& -R_{\text {owc } 1} f_{\text {owc } 1}+R_{o w c 2} f_{\text {owc } 2}
\end{aligned}\right.
$$

donde $d$ es el coeficiente de amortiguamiento equivalente, $M_{\text {wind }}$ y $M_{\text {wave }}$ son los momentos flectores causados por el viento externo y las cargas de las olas que actúan sobre la base de la torre y la parte superior de la plataforma, respectivamente. $F_{\text {owc } 1}$ y $F_{\text {owc } 2}$ son las fuerzas inducidas por las presiones en las cámaras de captura de OWC1 y OWC2, respectivamente.

Sustituyendo (3)-(5) en (1) y (2) y considerando aproximaciones de ángulo pequeño porque ninguna de las excede los 10 grados de inclinación, incluso en las cargas de viento y oleaje más fuertes y asumiendo que ambos OWC están a la misma altura distancia desde la bisagra de la torre $\left(\mathrm{R}_{\mathrm{OWC}}=\mathrm{R}_{\mathrm{OWC} 1}=\mathrm{R}_{\mathrm{OWC} 2}\right)$ el modelo dinámico no lineal de contacto de la turbina eólica flotante de barcaza se expresa por (6).

$$
\left\{\begin{array}{c}
I_{p} \ddot{\theta}_{p}-k_{t}\left(\theta_{t}-\theta_{p}\right)+m_{p} g R_{p} \theta_{p}+k_{p} \theta_{p}=-d_{p} \dot{\theta}_{p} \\
+d_{t}\left(\dot{\theta}_{t}-\dot{\theta}_{p}\right)+M_{\text {wave }}-R_{\text {owc }}\left(f_{\text {owc } 1}-f_{\text {owc } 2}\right) \\
I_{t} \ddot{\theta}_{t}+k_{t}\left(\theta_{t}-\theta_{p}\right)-m_{t} g R_{t} \theta_{t}=-d_{t}\left(\dot{\theta}_{t}-\dot{\theta}_{p}\right) \\
+M_{\text {wind }}
\end{array}\right.
$$

El viento y las olas interactúan con la estructura FOWT de una manera aeroelástica e hidroelástica compleja. Además, existe un acoplamiento inherente entre las respuestas estructurales inducidas por el viento y las olas [18].
Para obtener un modelo lineal, se asume que las cargas de viento y oleaje $M_{\text {wind }}$ y $M_{\text {wave }}$ se han obtenido linealmente por la velocidad del viento a la altura del eje $V_{\text {wind }}(t)$ y la elevación de la ola $Z(t)$, por lo que $M_{\text {wind }}$ y $M_{\text {wave }}$ están representadas con una dinámica de primer orden como:

$$
\begin{aligned}
& \dot{M}_{\text {wind }}(t)=-\alpha_{\text {wind }} M_{\text {wind }}(t)+\beta_{\text {wind }} V_{\text {wind }}(t) \\
& \dot{M}_{\text {wave }}(t)=-\alpha_{\text {wave }} M_{\text {wave }}(t)+\beta_{\text {wave }} Z(t)
\end{aligned}
$$

\subsection{LAS COLUMNAS DE AGUA OSCILANTES}

Suponiendo que la superficie libre interna dentro de la cámara del OWC se comporta como un pistón y la presión es uniforme, la fuerza se puede definir como $[19,20]$ :

$$
f_{\text {OWCi }}=-p_{i}(t) S \quad(i=1,2)
$$

donde pi $(\mathrm{t})$ es la presión de la cámara y $\mathrm{S}$ es la superficie libre interna de la cámara, que es la misma para ambos OWC. El subíndice $\mathrm{i}=1,2$ se refiere a OWC1 o OWC2.

Considerando el aire como un gas ideal y el sistema es adiabático y las transformaciones son lo suficientemente lentas como para ser reversibles, pueden entonces considerarse isentrópicas y la densidad del aire se define como:

$$
\rho_{i}(t)=\rho_{a}\left(\frac{p_{i}(t)}{p_{a}}\right)^{\frac{1}{\gamma}} \quad(i=1,2)
$$

donde $p_{a}$ y $\rho_{a}$ son la presión atmosférica y la densidad. $\gamma$ es la proporción de calor.

Al linealizar la relación isentrópica entre densidad y presión, obtenemos:

$$
\begin{array}{ll}
\rho_{i}(t)=\rho_{a}\left(\frac{p_{i}(t)}{p_{a} \gamma}\right) & (i=1,2) \\
\dot{\rho}_{i}(t)=\frac{\rho_{a}}{p_{a} \gamma} \dot{p}_{i}(t) & (i=1,2)
\end{array}
$$

El caudal de masa dentro de las cámaras se puede definir como:

$$
\dot{\rho}_{i}(t)=\frac{\rho_{a}}{p_{a} \gamma} \dot{p}_{i}(t) \quad(i=1,2)
$$

donde $V_{0}$ es el volumen de aire en la cámara y $V_{O W C}(t)$ es el volumen de aire instantáneo.

El volumen de aire oscilante VOWC (t) depende de la geometría de las cámaras de captura OWC y se define como:

$$
V_{O W C i}(t)=V_{0}-S Z_{1}(t)
$$

donde $S=l_{c} . w_{c}$ es la superficie libre interior de la cámara y $Z_{l}$ es el desplazamiento vertical del agua en forma de pistón, que aumenta en dirección ascendente. 
Por tanto, la presión dentro de las cámaras depende del caudal de masa y del volumen de aire oscilante:

$$
\dot{p}_{i}(t)=\frac{p_{a} \gamma}{\rho_{a} V_{0}} \dot{m}_{i}(t)-\frac{p_{a} \gamma}{V_{0}} \dot{V}_{i}(t) \quad(i=1,2)
$$

\section{CONTROL DE FLUJO DE AIRE COMPLEMENTARIO PROPUESTO}

Para ayudar a estabilizar el sistema FOWT, se han integrado dos $\mathrm{OWC}$ en la estructura de la plataforma de barcaza ITI Energy. Ambas OWC son idénticas y se han colocado a ambos lados de la torre (frontal y posterior) a la misma distancia para mantener la simetría. Al utilizar una estrategia de control de flujo para ajustar el flujo de aire y la presión dentro de las cámaras de captura, la OWC ayuda a oponerse a algunas de las fuerzas hidrodinámicas.

Debido a que las OWC están integrados en la plataforma barcaza ITI Energy y tienen momentos opuestos en ella, la estrategia de control de flujo de aire propuesta se basa en el paso de la plataforma como se explica en la Figura 2. En la práctica, la entrada al sistema FOWT es la elevación de la ola $Z(t)$ que se puede registrar la altura de ola en el centro de la plataforma con un Analizador de Corriente Doppler Acústico (ADCP) montado en la parte inferior de la barcaza. En cuanto a los DOF de FOWT, tanto el cabeceo de la plataforma $\theta_{p}$ como la escora superior de la torre $x_{t}$ pueden medirse con dos acelerómetros colocados en la base y la parte superior de la torre, respectivamente [1].

La estrategia de control propuesta utiliza dos controladores PID para abrir y cerrar la válvula de mariposa de acuerdo con el paso de la plataforma como se muestra en la Figura 2. La entrada de los controladores PID es el error de paso de la plataforma y la salida es la señal de control de las válvulas de aire. Cuando las válvulas atrapan o liberan el aire en las cámaras, aumentan o disminuyen la presión y consecuentemente las fuerzas que actúan sobre la barcaza del sistema FOWT.

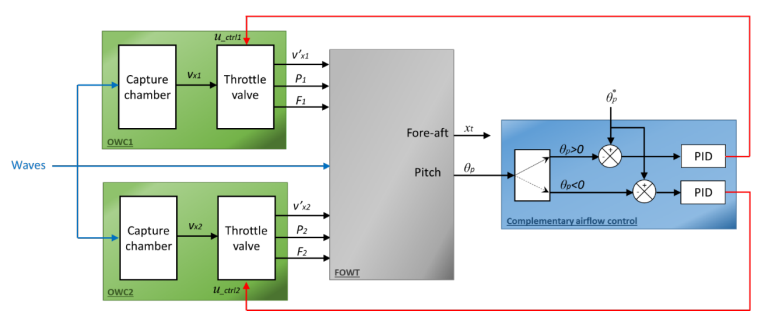

Figura 2: Control de flujo de aire complementario propuesto para la estabilización FOWT.

\section{RESULTADOS Y DISCUSIONES}

En este estudio, se comparan ambas estructuras para comprender su respuesta y cómo se amortiguan las perturbaciones sin ninguna excitación. En este sentido, no debe haber ninguna carga aerodinámica en el rotor y ninguna carga de olas en la estructura de soporte, por lo tanto, tanto la velocidad del viento a la altura del buje $V_{\text {wind }}(t)$ y la elevación de las olas $Z(t)$ deben ser omitido. A continuación, se proporciona una perturbación inicial al sistema en forma de un ángulo de inclinación inicial de la plataforma de 5 grados. Los resultados obtenidos se muestran en las Figuras 3 y 4.

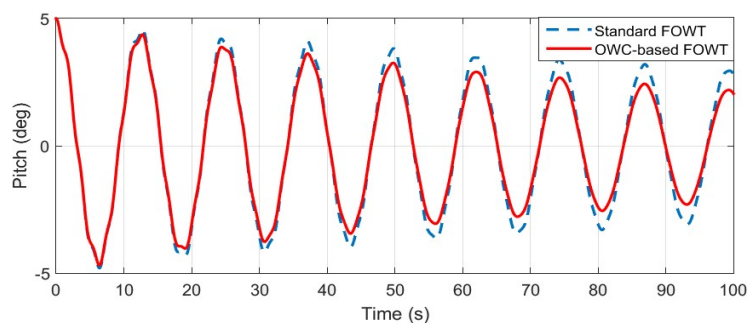

Figura 3: Respuesta de caída libre para el tono de la plataforma en FOWT estándar y FOWT basado en OWC.

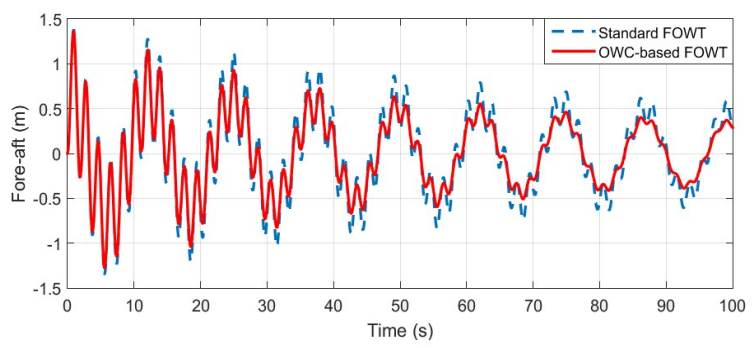

Figura 4: Respuesta de decaimiento libre para el desplazamiento longitudinal de la parte superior de la torre en el FOWT estándar y el FOWT basado en OWC.

La Figura 3 muestra la respuesta de amortiguación del cabeceo de la plataforma para ambas estructuras y se puede observar que ambas comienzan en 5 grados, que es la perturbación inicial, introducida en ambos sistemas. Se puede ver que, durante la simulación con el tiempo, el cabeceo de la plataforma se amortigua, pero con el FOWT propuesto basado en OWC, el cabeceo se reduce en comparación con el FOWT estándar.

En la Figura 4, se ilustra el desplazamiento longitudinal de la torre superior para ambas estructuras y se puede deducir el mismo; las oscilaciones del desplazamiento longitudinal se reducen en el FOWT basado en OWC en comparación con el FOWT estándar. 


\section{CONCLUSIONES}

En este documento, se han integrado columnas de agua oscilantes en la plataforma de tipo barcaza ITI Energy de una turbina eólica marina flotante y se ha propuesto un control de flujo de aire complementario para las OWC con el fin de ayudar a estabilizar la plataforma al reducir el movimiento de inclinación no deseado de la plataforma y la torre.

Para ello se ha desarrollado un modelo FOWT lineal simplificado que modela el cabeceo de la plataforma y la escora en la parte superior de la torre. A partir de este modelo se han integrado las presiones y fuerzas de los dispositivos OWC al objeto de estudiar la capacidad de las cámaras OWC para oponerse a las fuerzas hidrodinámicas que sufre la plataforma. Para ello se ha implementado un esquema de control que, a partir del ángulo de inclinación, establece la señal de control adecuada mediante el uso de controladores PID, que a su vez actúan sobre la válvulas de control de flujo de las cámaras OWC de manera que se regulan las presiones dentro de las cámaras.

Los resultados muestran que la respuesta de amortiguamiento del FOWT propuesto basado en sistemas OWC presenta mayor caída en comparación con la plataforma FOWT estándar basada en barcazas.

\section{Agradecimientos}

Este trabajo ha sido apoyado en parte por el Gobierno Vasco a través del proyecto IT1207-19 y por el MCIU/MINECO a través de los proyectos RTI2018094902-B-C21 y RTI2018-094902-B-C22 (MCIU / AEI / FEDER, UE).

\section{English summary}

\section{AIRFLOW CONTROL STRATEGY OF OSCILLATING WATER COLUMNS FOR FLOATING OFFSHORE WIND TURBINE STABILIZATION}

\author{
Abstract \\ This paper presents the idea of incorporating \\ oscillating water columns (OWCs) into the barge \\ platform of a floating offshore wind turbines (FOWT) \\ to make it a multi-purpose platform for harnessing \\ both wind and wave energies. Moreover, the OWCs \\ will help stabilize the FOWT platform by means of an \\ airflow control strategy used to reduce the platform
}

pitch and tower top fore-aft displacement. This is achieved by a proposed airflow control strategy to control the valves within the OWCs. The comparative study between a standard FOWT and the proposed OWC-based FOWT shows an improvement in the platform's stability.

Keywords: airflow control; barge platform; floating offshore wind turbine; oscillating water column; wave energy; wind energy.

\section{Referencias}

[1] Hu, J., Zhou, B., Vogel, C., Liu, P., Willden, R., Sun, K., Zang, J., Geng, J., Jin, P., Cui, L. and Jiang, B., (2020) "Optimal design and performance analysis of a hybrid system combing a floating wind platform and wave energy converters", Applied Energy, 269, p.114998.

[2] Sarmiento, J., Iturrioz, A., Ayllón, V., Guanche, R. and Losada, I.J., (2019) "Experimental modelling of a multi-use floating platform for wave and wind energy harvesting", Ocean Engineering, 173, pp.761-773.

[3] Yu, J., Li, Z., Yu, Y., Hao, S., Fu, Y., Cui, Y., $\mathrm{Xu}, \mathrm{L}$. and $\mathrm{Wu}, \mathrm{H}$. , (2020) "Design and Performance Assessment of Multi-Use Offshore Tension Leg Platform Equipped with an Embedded Wave Energy Converter System", Energies, 13(15), p.3991.

[4] Kluger, J.M., Slocum, A.H. and Sapsis, T.P., 2017, July. "A first-order dynamics and cost comparison of wave energy converters combined with floating wind turbines", In The 27th International Ocean and Polar Engineering Conference. International Society of Offshore and Polar Engineers.

[5] Slocum, A., Kluger, J. and Mannai, S., 2019, July. "Energy Harvesting and Storage System Stabilized Offshore Wind Turbines", In 2019 Offshore Energy and Storage Summit (OSES) (pp. 1-6).

[6] Kamarlouei, M., Gaspar, J.F., Calvário, M., Hallak, T.S., Mendes, M.J., Thiebaut, F. and Soares, C.G., (2020) "Experimental analysis of wave energy converters concentrically attached on a floating offshore platform", Renewable Energy, 152, pp.1171-1185.

[7] Vijfhuizen, W.J.M.J. 2006, September. "Design of a Wind and Wave Power Barge", Master's Dissertation, Department of Naval Architecture and Mechanical Engineering, Universities of Glasgow and Strathclyde, Glasgow, Scotland. 
[8] M'zoughi, F., Garrido, I., Bouallègue, S., Ayadi, M. and Garrido, A.J., (2019) "Intelligent Airflow Controls for a Stalling-Free Operation of an Oscillating Water Column-Based Wave Power Generation Plant", Electronics, 8(1), p.70.

[9] M'zoughi, F., Garrido, I., Garrido, A.J. and De La Sen, M., (2020) "ANN-based airflow control for an oscillating water column using surface elevation measurements", Sensors, 20(5), p.1352.

[10] M'zoughi, F., Garrido, I., Garrido, A.J. and De La Sen, M., (2020) "Self-adaptive global-best harmony search algorithm-based airflow control of a wells-turbine-based oscillating-water column”, Applied Sciences, 10(13), p.4628.

[11] Jonkman, J.M., (2007) "Dynamics modeling and loads analysis of an offshore floating wind turbine", (No. NREL/TP-500-41958). National Renewable Energy Lab.(NREL), Golden, CO (United States).

[12] Jonkman, J., 2008, January. "Influence of control on the pitch damping of a floating wind turbine", In 46th AIAA Aerospace Sciences Meeting and Exhibit (p. 1306).

[13] Lackner, M.A. and Rotea, M.A., (2011) "Passive structural control of offshore wind turbines", Wind energy, 14(3), pp.373-388.

[14] Stewart, G. and Lackner, M., (2013) “Offshore wind turbine load reduction employing optimal passive tuned mass damping systems", IEEE transactions on control systems technology, 21(4), pp.1090-1104.

[15] Si, Y. and Karimi, H.R., (2014) "Gain scheduling $\mathrm{H} 2 / \mathrm{H} \infty$ structural control of a floating wind turbine", IFAC Proceedings Volumes, 47(3), pp.6788-6793.
[16] Lackner, M.A. and Rotea, M.A., (2011) "Structural control of floating wind turbines", Mechatronics, 21(4), pp.704-719.

[17] Aboutalebi, P., M'zoughi, F., Garrido, I. and Garrido, A.J., (2021) "Performance Analysis on the Use of Oscillating Water Column in BargeBased Floating Offshore Wind Turbines", Mathematics, 9(5), p.475.

[18] Hu, Y., Wang, J., Chen, M.Z., Li, Z. and Sun, Y., (2018) "Load mitigation for a barge-type floating offshore wind turbine via inerter-based passive structural control", Engineering Structures, 177, pp.198-209.

[19] Aubault, A., Alves, M., Sarmento, A.N., Roddier, D. and Peiffer, A., 2011, January. "Modeling of an oscillating water column on the floating foundation WindFloat", In International Conference on Offshore Mechanics and Arctic Engineering (Vol. 44373, pp. 235-246).

[20] Henriques, J.C.C., Sheng, W., Falcão, A. and Gato, L.M.C., 2017, June. "A comparison of biradial and wells air turbines on the Mutriku breakwater OWC wave power plant", In $A S M E$ 2017 36th International Conference on Ocean, Offshore and Arctic Engineering. American Society of Mechanical Engineers Digital Collection.

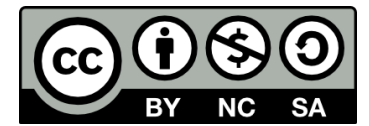

(C) 2021 by the authors. Submitted for possible open access publication under the terms and conditions of the Creative Commons Attribution CC BY-NC-SA 4.0 license (https://creativecommons.org/licenses/byncsa/4.0/deed.es). 